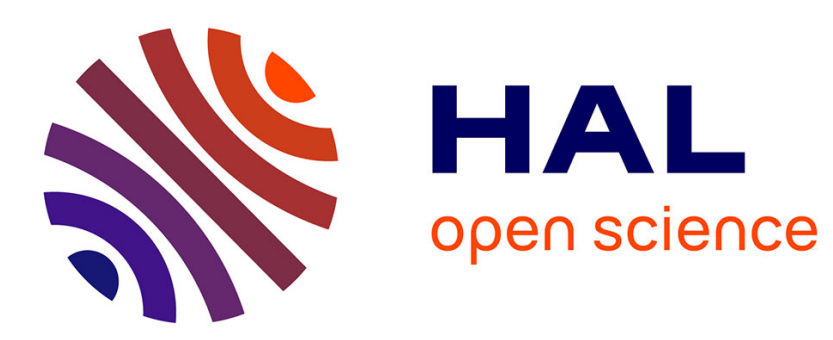

\title{
La Théorie Variationnelle des Rayons Complexes pour le calcul des vibrations moyennes fréquences
}

Pierre Ladevèze, Lionel Arnaud, Philippe Rouch, Claude Blanzé

\section{To cite this version:}

Pierre Ladevèze, Lionel Arnaud, Philippe Rouch, Claude Blanzé. La Théorie Variationnelle des Rayons Complexes pour le calcul des vibrations moyennes fréquences. Revue Européenne des Éléments Finis, 2000, 9 (1-3), pp.67-88. 10.1080/12506559.2000.10511430 . hal-01465391

\author{
HAL Id: hal-01465391 \\ https://hal.science/hal-01465391
}

Submitted on 24 Feb 2017

HAL is a multi-disciplinary open access archive for the deposit and dissemination of scientific research documents, whether they are published or not. The documents may come from teaching and research institutions in France or abroad, or from public or private research centers.
L'archive ouverte pluridisciplinaire HAL, est destinée au dépôt et à la diffusion de documents scientifiques de niveau recherche, publiés ou non, émanant des établissements d'enseignement et de recherche français ou étrangers, des laboratoires publics ou privés.

\section{(c)(1)}

Distributed under a Creative Commons Attribution| 4.0 International License 


\title{
La Théorie Variationnelle des Rayons Complexes pour le calcul des vibrations moyennes fréquences
}

\author{
Pierre Ladevèze - Lionel Arnaud \\ Philippe Rouch - Claude Blanzé
}

Laboratoire de Mécanique et Technologie, ENS Cachan/CNRS/Université Paris 6 61, avenue du Président Wilson, F-94235 Cachan cedex [ladeveze, arnaud, rouch, blanze]@lmt.ens-cachan.fr

RÉSUME. Une nouvelle approche, appelée "Théorie Variationnelle des Rayons Complexes 》, est développée pour calculer les vibrations de structures élastiques faiblement amorties dans le domaine des moyennes fréquences. Les grandeurs effectives (énergie de déformation, intensité vibratoire...) sont évaluées suite à la résolution d'un système d'équations de faible dimension qui, de près comme de loin, $n$ 'est pas issu d'une discrétisation «éléments finis » fine de la structure. L'accent est mis, à travers un certain nombre d'exemples, sur l'intérêt et les possibilités de cette nouvelle approche.

ABSTRACT. A new approach named the "Variational Theory of Complex Rays" is introduced for computing the vibrations of elastic structures weakly damped in the medium frequency range. Emphasis has been placed here on the most fundamental aspects. The effective quantities (elastic energy, vibration intensity...) are evaluated after computing a small system of equations which does not derive from a finite element dicretization of the structure. Numerical examples related to plates show the interest and the possibilities of the VTRC.

MOTS-CLES : vibrations, moyennes fréquences, plaque, rayons complexes

KEY WORDS : vibrations, medium frequency, plate, complex rays 


\section{Introduction}

La modélisation et le calcul des structures élastiques en vibration constituent sans aucun doute un aspect majeur de la conception des structures. Le domaine des basses fréquences ne pose pas de difficultés majeures, même pour les structures complexes, au moins pour la modélisation et le calcul. Dans le domaine des hautes fréquences, il existe des outils de calcul qui sont assez distincts de ceux utilisés pour les basses fréquences - en particulier la méthode S.E.A.(Statistical Energy Analysis), pour laquelle l'aspect spatial de la structure disparaît presque entièrement [LYO 67] [MAC 94] [DOW 85].

En revanche, la modélisation et le calcul des vibrations moyennes fréquences, objet de cet article, posent encore des problèmes. La difficulté vient de la longueur d'onde des phénomènes étudiés, très petite par rapport à la dimension caractéristique de la structure. L'extension des méthodes basses fréquences, basées sur une discrétisation de type «éléments finis » (E.F.) conduit à un nombre de degrés de liberté trop élevé avec, de plus, de sérieux obstacles numériques.

De nombreux travaux sont en cours pour étendre le domaine fréquentiel calculable. Un premier résultat est relatif à l'erreur apparaissant lors de calculs E.F. ; l'erreur de pollution conduit à des résultats dont la qualité est inférieure à celle attendue [ILH 95] [ILH 97] [BOU 98] [DER 99]. De nombreuses améliorations basées sur des méthodes E.F. ont été explorées, en particulier: [BAB 95] [HAR 96] [LAN 91] [BAR 98] [WIB 96] [DEM 92] [GRE 99] [GRO 98] [WU 98] [ZIE 87] [LIU 91] [LEU 62] [RIZ 85] [ROS 97].

L'extension aux moyennes fréquences des méthodes issues de la S.E.A. rencontre également d'importantes difficultés.

Peu de travaux ont pour objectif le calcul des vibrations dans le domaine spécifique des moyennes fréquences. Pour les barres et les poutres, le problème est plus simple et a été résolu dans : [CUS 90] [GIR 93] [NEF 89]. En revanche, très peu de travaux sont relatifs aux plaques et aux coques: [MOR 92] [LAN 91] [SOI 85] [SOI 98] [OHA 89]; fortement liées aux E.F, ces méthodes introduisent des bases réduites spécifiques, en espace et en fréquence, dans le domaine des vibrations moyennes fréquences.

A l'exception de la théorie introduite par [BEL 75], ces méthodes ne sont pas, pour nous, de véritables théories moyennes fréquences, car les phénomènes à petite longueur de variation, dont le détail n'a pas de sens physique, sont toujours présents. Elles ne font pas réellement intervenir les quantités effectives pour les échelles de temps et d'espace considérées et donnent toujours des résultats très sensibles aux petites variations de données. La théorie introduite initialement par [BEL 75] [BEL 77] et [BUV 80] est construite sur les notions de densité d'énergie effective et de flux d'énergie effectif. Cette théorie heuristique est très séduisante. Cependant, malgré les améliorations apportées par [LAS 94] [ICH 97] [LUZ 94], elle contient toujours des obstacles difficiles à contourner [LAD 95].

L'approche proposée ici, appelée " Théorie Variationnelle des Rayons Complexes » (T.V.R.C.), introduite dans [LAD 96a] [LAD 96b], constitue une véritable alternative pour le calcul des vibrations moyennes fréquences. L'objectif de ce premier article est 
de présenter les points fondamentaux de la T.V.R.C. et d'illustrer ses possibilités et ses limites à l'aide d'exemples numériques relatifs aux plaques.

Pour simplifier cette présentation, nous considérerons seulement le problème des vibrations forcées, à une fréquence fixe, sur un problème modèle d'élasticité $2 \mathrm{D}$.

\section{Le problème de référence décrivant les vibrations moyennes fréquences}

Le problème de référence que nous introduisons peut être qualifié de modèle : tout ce que nous faisons et dirons peut être étendu aux différents problèmes qui se posent dans la pratique, problèmes qui mettent en jeu des poutres, des plaques, des coques et des milieux 3D. Il s'agit d'un problème plan qui décrit, par exemple, les vibrations dans la section droite d'une structure cylindrique extrêmement raide et bloquée dans la direction axiale. Aussi, toutes les quantités que nous introduisons sont planes; ce sont des amplitudes, définies sur le corps des complexes, qui dépendent de la fréquence d'excitation $\omega$. Ainsi, à l'amplitude $\underline{Q}(\underline{X})$, on associe $\underline{Q}(\underline{X}) \exp (i \omega t)$.

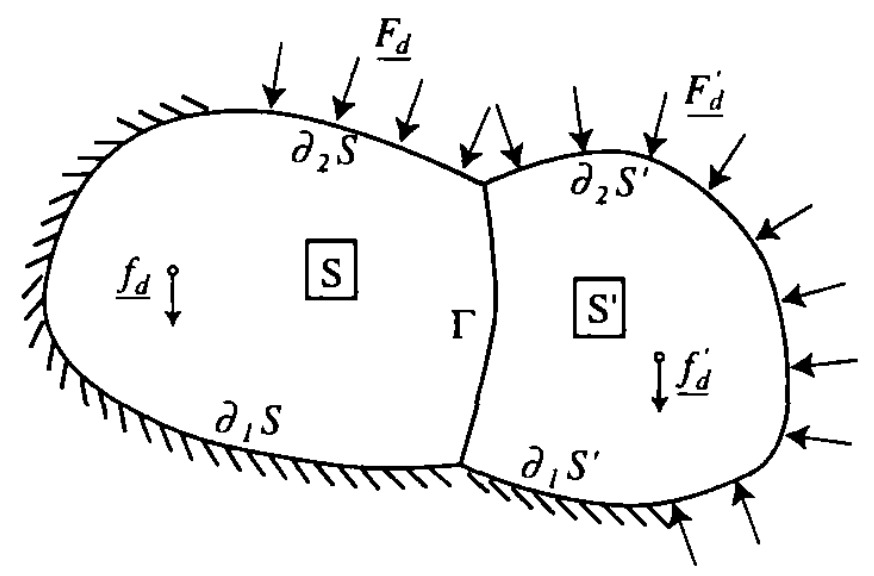

Figure 1. Problème de référence

Le problème de référence décrit les vibrations pour une excitation de fréquence fixée. Plaçons-nous dans la situation où la structure étudiée est l'assemblage de deux sous-structures homogènes $S$ et $S^{\prime}$ [Fig.1]. Toutes les quantités relatives à $S^{\prime}$ seront affectées de " ' ». Le déplacement $\underline{U}$ (resp. $\underline{U}^{\prime}$ ) et la contrainte $\sigma$ (resp. $\left.\sigma^{\prime}\right)$ sont supposés à énergie finie sur $S$ (resp. $S^{\prime}$ ); les espaces correspondants sont $\mathcal{U}$ et $\mathcal{S}$ (resp. $\mathcal{U}^{\prime}$ et $\mathcal{S}^{\prime}$ ). La structure est dans un environnement connu :

— un déplacement $\underline{U}_{d}$ (resp. $\underline{U}_{d}^{\prime}$ ) sur la partie $\partial_{1} S$ (resp. $\partial_{1} S^{\prime}$ ) de la frontière de $S$ (resp. $S^{\prime}$ )

- une densité d'effort $\underline{F_{d}}$ (resp. $F_{d}^{\prime}$ ) sur la partie $\partial_{2} S$ (resp. $\partial_{2} S^{\prime}$ ) complémentaire de $\partial_{1} S$ (resp. $\partial_{1} S^{\prime}$ ),

— une densité d'effort $\underline{f_{d}}$ (resp. $\underline{f_{d}^{\prime}}$ ) sur la section $S$ (resp. $S^{\prime}$ ). Introduisons pour la sous-structure $S$, l'espace $\mathbf{S}_{\mathbf{a d}}$ des couples déplacement- contrainte 
$s=(\underline{U}, \sigma)$ tels que :

$$
\begin{aligned}
& \underline{U} \in \mathcal{U} \quad \sigma \in \mathcal{S} \\
& \underline{\Gamma}=\underline{d i v} \sigma+\underline{f d}_{d} \operatorname{sur} S \\
& \sigma=(1+i \eta) \overline{\mathbf{K}}_{\mathbb{E}}(\underline{U}) \quad \text { et } \quad \underline{\Gamma}=-\omega^{2} \rho(1-i \theta) \underline{U}
\end{aligned}
$$

Les notations sont usuelles. $\mathbf{K}$ est l'opérateur de Hooke et $\rho$ la densité. $\eta$ et $\theta$ sont des coefficients d'amortissement caractéristiques du matériau; ils dépendent de $\omega$. Le sous-espace $\mathbf{S}=\mathcal{U} \times \mathcal{S}$ associé aux conditions homogènes $\left(f_{d}=0\right)$ est désigné par $\mathbf{S}_{\mathbf{a d}, \mathbf{0}}$. Pour la sous-structure $S^{\prime}$, on introduit de la même façon $\mathbf{S}_{\mathbf{a d}}^{\prime}$ et $\mathbf{S}_{\mathbf{a d}, \mathbf{0}}^{\prime}$.

Le problème de référence s'écrit alors :

Trouver $(\underline{U}(\underline{X}), \sigma(\underline{X}) ; \underline{X} \in S)$ et $\left(\underline{U^{\prime}}(\underline{X}), \sigma^{\prime}(\underline{X}) ; \underline{X} \in S^{\prime}\right)$ tels que :

$$
\begin{array}{ll}
s=(\underline{U}, \sigma) \in \mathbf{S}_{\mathbf{a d}} & s^{\prime}=\left(\underline{U}^{\prime}, \sigma^{\prime}\right) \in \mathbf{S}_{\mathbf{a d}}^{\prime} \\
\left.\underline{U}\right|_{\partial_{1} S}=\underline{U}_{d} & \left.\sigma \underline{\underline{n}}\right|_{\partial_{2} S}=\underline{F}_{d} \\
\underline{U^{\prime}} \mid \partial_{1} S^{\prime}=\underline{U}_{d}^{\prime} & \left.\sigma^{\prime} \underline{\underline{n}}^{\prime}\right|_{\partial_{2} S^{\prime}}=F_{d}^{\prime} \\
\left.\left(\underline{U}-\underline{U^{\prime}}\right)\right|_{\Gamma}=0 & \left.\left(\sigma \underline{n}-\sigma^{\prime} \underline{n}^{\prime}\right)\right|_{\Gamma}=0 \\
\text { (condition de transmission à la traversée de } \Gamma) &
\end{array}
$$

$\underline{n}$ (resp. $\left.\underline{n}^{\prime}\right)$ est la normale extérieure à la frontière de $S$ (resp. $\left.S^{\prime}\right)$.

\section{REMARQUES.}

— Les termes d'amortissement, qui sont introduits ici, sont associés à $\theta$ et $\eta$. Ils conduisent à des matrices d'amortissement identiques à celles utilisées couramment dans la pratique. En effet, suite à une discrétisation E.F., on obtient par exemple pour la sous- structure $S$, que la matrice d'amortissement $\mathbf{B}$ est une combinaison des matrices de masse et de rigidité :

$$
\mathbf{B}=\frac{1}{\omega}\left(\omega^{2} \theta \mathbf{M}+\eta \mathbf{K}\right)
$$

- Il est aisé de montrer que le problème de référence admet au plus une solution si :

- les opérateurs de Hooke $\mathbf{K}$ et $\mathbf{K}^{\prime}$ sont définis positifs,

— les coefficients d'amortissement $\theta, \theta^{\prime}, \eta, \eta^{\prime}$ sont positifs ou nuls.

\section{Nouvelle formulation variationnelle}

Cette formulation traduit les conditions aux limites tant en déplacement qu'en effort, en particulier les conditions de transmission à la traversée de $\Gamma$. C'est le premier principe (P1) de la Théorie Variationnelle des Rayons Complexes. Aussi, et c'est un point fondamental, aucun lien n'est introduit a priori entre $s=(\underline{U}, \sigma)$ et $s^{\prime}=\left(\underline{U^{\prime}}, \sigma^{\prime}\right)$. Cette formulation s'écrit : 
Trouver $s=(\underline{U}, \sigma) \in \mathbf{S}_{\mathbf{a d}}$ et $s^{\prime}=\left(\underline{U^{\prime}}, \sigma^{\prime}\right) \in \mathbf{S}_{\mathbf{a d}}^{\prime}$ tels que :

$$
\begin{array}{r}
\mathcal{R}_{e}\left\{-i \omega\left[\int_{\partial_{1} S} \delta \sigma \underline{n} \circ\left(\underline{U}^{*}-\underline{U}_{d}^{*}\right) d \tau+\int_{\partial_{2} S}\left(\sigma \underline{n}-\underline{F}_{d}\right) \circ \delta \underline{U}^{*} d \tau+\right.\right. \\
\int_{\partial_{1} S^{\prime}} \delta \sigma^{\prime} \underline{n}^{\prime} \circ\left(\underline{U^{\prime *}}-{\underline{U^{\prime}}}_{d}^{*}\right) d \tau^{\prime}+\int_{\partial_{2} S^{\prime}}\left(\sigma^{\prime} \underline{n}^{\prime}-\underline{F}_{d}^{\prime}\right) \circ \delta{\underline{U^{\prime}}}^{*} d \tau^{\prime}+ \\
\left.\left.\int_{\Gamma} \frac{1}{2}\left[\left(\delta \sigma \underline{n}-\delta \sigma^{\prime} \underline{n}^{\prime}\right) \circ\left(\underline{U}^{*}-{\underline{U^{\prime}}}^{*}\right)+\left(\sigma \underline{n}+\sigma^{\prime} \underline{n}^{\prime}\right) \circ\left(\delta \underline{U}^{*}+\delta \underline{U}^{\prime *}\right)\right] d \tau\right]\right\}=0 \\
\forall \delta s \in \mathbf{S}_{\mathbf{a d}, \mathbf{0}} \text { et } \forall \delta s^{\prime} \in \mathbf{S}_{\mathbf{a d}, \mathbf{0}}^{\prime}
\end{array}
$$

$\mathcal{R}_{e}$ désigne la partie réelle; $\underline{U}^{*}$ est le conjugué de $\underline{U}$. Cette nouvelle formulation du problème initial est équivalente au problème initial sous certaines conditions. Précisément, on a :

Théorème 1. La nouvelle formulation est équivalente au problème de référence [2] si :

- le problème de référence [1] admet une solution,

- les conditions [3] sur les caractéristiques des matériaux sont vérifiées.

\section{REMARQUES.}

- Les quantités qui interviennent dans la nouvelle formulation sont des puissances. Cette formulation est une extension aux problèmes de dynamique de la formulation proposée dans [LAD 83] pour traiter les problèmes statiques et qui depuis a été appliquée dans différents domaines. Au niveau des conditions de transmission entre plusieurs sous-structures, c'est la variante due à Hochard [HOC 93] qui est étendue ici.

- La nouvelle formulation, contrairement à la formulation classique, a un sens pour les problèmes mal posés. Par exemple, on peut imposer sur une même partie du bord à la fois le déplacement et la distribution d'effort; le terme correspondant est alors :

$$
\frac{1}{2} \mathcal{R}_{e}\left\{-i \omega\left[\int_{L} \delta \sigma \underline{n} \circ\left(\underline{U}^{*}-\underline{U}_{d}^{*}\right) d \tau+\left(\sigma \underline{n}-\underline{F}_{d}\right) \circ \delta \underline{U}^{*}\right] d \tau\right\}
$$

- L'amortissement joue un rôle majeur dans la nouvelle formulation ; introduisons pour la sous-structure $S$, la puissance dissipée due à l'amortissement qui, par convention, est :

$$
E_{D}(\underline{U})=\frac{\omega^{3}}{2} \int_{S} \rho \theta \underline{U} \circ \underline{U}^{*} d S+\frac{\omega}{2} \int_{S} \eta \operatorname{Tr}\left[\mathbf{K} \varepsilon(\underline{U}) \varepsilon\left(\underline{U}^{*}\right)\right] d S
$$

Pour $S^{\prime}$, on définit de la même façon $E_{D}^{\prime}\left(\underline{U}^{\prime}\right)$. Il est aisé de voir que la nouvelle formulation peut se mettre sous la forme:

Trouver $s \in \mathbf{S}_{\mathbf{a d}}$ et $s^{\prime} \in \mathbf{S}_{\mathbf{a d}}^{\prime}$ tels que : 


$$
\begin{gathered}
\delta\left[E_{D}(\underline{U})+E_{D}^{\prime}\left(\underline{U}^{\prime}\right)\right]+<\left[\begin{array}{c}
s \\
s^{\prime}
\end{array}\right], \delta\left[\begin{array}{c}
s \\
s^{\prime}
\end{array}\right]>=\left(L_{d}, \delta\left[\begin{array}{c}
s \\
s^{\prime}
\end{array}\right]\right) \\
\forall \delta s \in \mathbf{S}_{\mathbf{a d}, \mathbf{0}} \quad \forall \delta s^{\prime} \in \mathbf{S}_{\mathbf{a d}, \mathbf{0}}^{\prime}
\end{gathered}
$$

où $L_{d}$ est une forme linéaire dépendant des données. La forme bilinéaire $<\bullet, \bullet>$ est définie en fait sur les frontières des sous-structures. Sa principale propriété est :

$$
<u, v>=-<v^{*}, u^{*}>
$$

La partie symétrique de l'opérateur linéaire associé à la nouvelle formulation dépend directement de l'amortissement. Au niveau discrétisé, il lui correspondra la matrice d'amortissement.

- Une conséquence de [8] et [7] est que la puissance dissipée est égale à :

$$
E_{D}(\underline{U})+E_{D}^{\prime}\left(\underline{U}^{\prime}\right)=\frac{1}{2}\left(L_{d},\left[\begin{array}{c}
s \\
s^{\prime}
\end{array}\right]\right)
$$

\section{Approximation}

\subsection{Principe}

Pour élaborer des approximations à partir de la nouvelle formulation, il suffit de définir des sous-espaces $\mathbf{S}_{\mathbf{a d}}^{\mathbf{h}}$ et $\mathbf{S}_{\mathbf{a d , 0}}^{\mathbf{h}}$ (resp. $\mathbf{S}_{\mathbf{a d}}^{\mathbf{h}}$ et $\mathbf{S}_{\mathbf{a d}, 0}^{\mathbf{h}}$ ) de $\mathbf{S}_{\mathbf{a d}}$ et $\mathbf{S}_{\mathbf{a d}, 0}$ (resp. $\mathbf{S}_{\mathbf{a d}}^{\prime}$ et $\mathbf{S}_{\mathbf{a d}, 0}^{\prime}$ ). Le problème approché correspondant admet une solution unique sous les conditions [3]; il s'écrit :

$$
\begin{aligned}
& \text { Trouver } s^{h}=(\underline{U}, \sigma) \in \mathbf{S}_{\mathbf{a d}}^{\mathbf{h}} \text { et } s^{\prime h}=\left(\underline{U^{\prime}}, \sigma^{\prime}\right) \in \mathbf{S}_{\mathbf{a d}}^{\mathbf{h}} \text { tels que : } \\
& \delta\left[E_{D}\left(\underline{U}^{h}\right)+E_{D}^{\prime}\left(\underline{U}^{\prime h}\right)\right]+<\left[\begin{array}{c}
s^{h} \\
s^{\prime h}
\end{array}\right], \delta\left[\begin{array}{c}
s^{h} \\
s^{\prime h}
\end{array}\right]>=\left(L_{d}, \delta\left[\begin{array}{c}
s^{h} \\
s^{\prime h}
\end{array}\right]\right) \\
& \forall \delta s^{h} \in \mathbf{S}_{\mathbf{a d}, 0}^{\mathbf{h}} \forall \delta s^{\prime h} \in \mathbf{S}_{\mathbf{a d}, 0}^{\mathbf{h}}
\end{aligned}
$$

Le second principe (P2) caractérisant la Théorie Variationnelle des Rayons Complexes est dans la construction du sous-espace $\mathbf{S}_{\mathbf{a d}}^{\mathbf{h}}$ associé à la sous-structure $S$. L'idée est d'introduire des approximations à deux échelles de fort contenu mécanique en distinguant trois zones: la zone intérieure, les zones «bords » et les zones « coins». Par exemple, au voisinage d'un point de la zone intérieure, la solution est supposée bien décrite par la superposition d'un nombre infini de modes locaux de vibration qui s'écrivent en introduisant une variable lente $\underline{X}$ et une variable rapide $\underline{Y}$ : Avec $\underline{X}=\underline{Y}$ :

$$
\begin{aligned}
& \underline{U}(\underline{X}, \underline{Y}, \underline{P})=\underline{W}(\underline{X}, \underline{Y}, \underline{P}) \exp (i \omega \underline{P} \circ \underline{Y}) \\
& \underline{\sigma}(\underline{X}, \underline{Y}, \underline{P})=\mathbf{C}(\underline{X}, \underline{Y}, \underline{P}) \exp (i \omega \underline{P} \circ \underline{Y})
\end{aligned}
$$

où $\underline{P}$ est un vecteur caractérisant le mode local de vibration. Les modes satisfont aux équations de la dynamique en milieu infini. Une caractéristique majeure de ces modes 
de base est d'être à deux échelles; nous verrons qu'ils sont définis explicitement en fonction de la variable rapide et en conséquence les paramètres qui deviendront les inconnues du problème à résoudre ne dépendent que de la variable lente. Pour une zone «bord », la construction des modes de base est voisine de la précédente, excepté qu'ils satisfont aux équations de la dynamique en milieu semi-infini. Pour une zone " coin », la démarche est similaire.

\subsection{Rayons complexes d'ordre $n$ relatifs à la zone intérieure}

Considérons la sous-structure $S$ supposée homogène. Pour simplifier, plaçonsnous dans le cas où les forces volumiques sont nulles $\left(f_{d}=0\right)$. La première caractéristique d'un rayon d'ordre $n$ relatif à la zone intérieure est d'être un mode de vibration du milieu infini de mêmes caractéristiques que la sous-structure $S$ et donc d'appartenir à $\mathbf{S}_{\mathbf{a d}}$. On a :

$$
\begin{aligned}
& -k^{2} \rho \omega^{2} \underline{U}=\underline{\operatorname{div}}(\mathbf{K} \varepsilon(\underline{U})) \quad k^{2}=(1-i \theta) /(1+i \eta) \\
& \sigma=(1+i \eta) \mathbf{K} \varepsilon(\underline{U})
\end{aligned}
$$

Les amortissements sont supposés faibles; on prendra donc $k \sim 1-i \frac{\delta}{2}$ avec $\delta=\theta+\eta$. La seconde caractéristique d'un rayon complexe d'ordre $n$ tient à son expression:

$$
\begin{aligned}
\underline{U}(\underline{X}, \underline{Y}, \underline{P}) & =\underline{W}(\underline{X}, \underline{P}) \exp \left(\frac{\delta}{2} \omega \underline{P} \circ \underline{X}\right) \exp (i \omega \underline{P} \circ \underline{Y}) \\
\sigma(\underline{X}, \underline{Y}, \underline{P}) & =(1+i \eta) \mathbf{K} \varepsilon(\underline{U})
\end{aligned}
$$

où on impose que l'amplitude soit un polynôme de degré $n$ en la variable lente $\underline{X}$.

REMARQUE. - Les rayons complexes d'ordre 0 peuvent être considérés comme le premier terme d'un développement asymptotique où le petit paramètre est l'inverse de la fréquence $(1 / \omega)$. Il s'agit de la notion bien connue de rayon, à la base de l'optique géométrique, notion qui a été fort étudiée [BOU 94] et [OHA 89]. Ici, cette démarche est revisitée pour construire des solutions exactes en milieu infini.

\subsubsection{Rayons complexes d'ordre 0}

Un rayon complexe d'ordre 0 est de la forme :

$$
\begin{aligned}
\underline{U}(\underline{X}, \underline{Y}, \underline{P}) & =\underline{W}(\underline{P}) \exp \left(\frac{\delta}{2} \omega \underline{P} \circ \underline{X}\right) \exp (i \omega \underline{P} \circ \underline{Y}) \\
\sigma(\underline{X}, \underline{Y}, \underline{P}) & =(1+i \eta) \mathbf{K} \varepsilon(\underline{U})
\end{aligned}
$$

Des équations [11], on tire que $(\underline{U}, \sigma) \in \mathbf{S}_{\mathbf{a d}}$ si et seulement si :

$$
\underline{W}=\frac{1}{\rho} \mathbf{K}\left[\underline{W} \underline{P}^{t}\right]_{s y m} \underline{P}
$$


En posant $\mathbf{A} \bullet=\frac{1}{\rho} \mathbf{K}\left[\bullet \underline{u}^{t}\right]_{s y m} \underline{u}$ avec $\underline{u}=\frac{P}{|\underline{P}|}$, le problème précédent, pour une direction $\underline{u}$ donnée, se ramène au problème aux valeurs propres:

$$
\text { Trouver }(p, \underline{W} \neq 0) \text { tel que } \underline{W}=p^{2} \mathbf{A} \underline{W}
$$

Propriété 1. L'opérateur A est symétrique et défini positif si les caractéristiques du matériau vérifient les conditions [3].

La théorie spectrale classique s'applique ; il existe pour toute direction $\underline{u}$, deux couples $\left(p_{1}, \underline{W}_{1}\right)$ et $\left(p_{2}, \underline{W}_{2}\right)$ solutions de [14] avec :

$$
p_{2} \geq p_{1} \geq 0 \quad\left\|\underline{W}_{1}\right\|=\left\|\underline{W}_{2}\right\|=1 \quad \underline{W}_{1} \circ \underline{W}_{2}=0
$$

P appartient à l'une des deux courbes $\mathcal{C}_{1}$ et $\mathcal{C}_{2}$ caractéristiques du matériau :

$$
\mathcal{C}_{1}=\left\{\underline{P} \mid \underline{P}=p_{1} \underline{u},\|\underline{u}\|=1\right\}, \quad \mathcal{C}_{2}=\left\{\underline{P} \mid \underline{P}=p_{2} \underline{u},\|\underline{u}\|=1\right\}
$$

Exemple : Pour un matériau isotrope, l'opérateur de Hooke s'écrit :

$$
\mathbf{K} \varepsilon=\gamma \operatorname{Tr}[\varepsilon] \mathbf{I}_{\mathbf{d}}+2 \mu \notin
$$

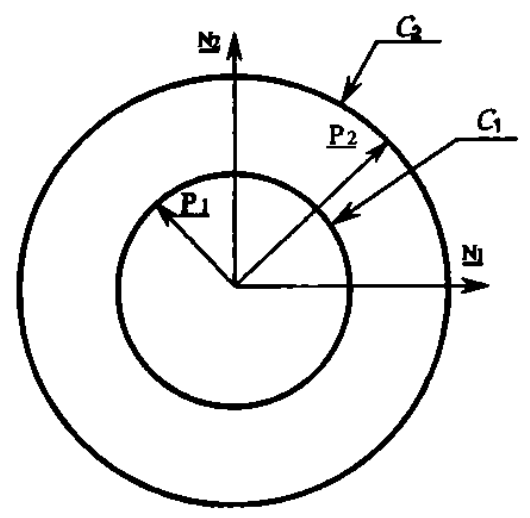

Figure 2. Ensemble des vecteurs d'onde admissibles en zone intérieure

$\mathcal{C}_{1}$ et $\mathcal{C}_{2}$ sont des cercles respectivement de rayons $\left[\frac{\rho}{\mu}\right]^{1 / 2}$ et $\left[\frac{\rho}{2 \mu+\gamma}\right]^{1 / 2}[$ Fig.2].

\subsubsection{Rayons complexes d'ordre $n$ - Construction}

Rappelons qu'ils se mettent sous la forme :

$$
\begin{aligned}
\underline{U}(\underline{X}, \underline{Y}, \underline{P}) & =\underline{W}(\underline{X}, \underline{P}) \exp \left(\frac{\delta}{2} \omega \underline{P} \circ \underline{X}\right) \exp (i \omega \underline{P} \circ \underline{Y}) \\
\sigma(\underline{X}, \underline{Y}, \underline{P}) & =(1+i \eta) \mathbf{K} \varepsilon(\underline{U})
\end{aligned}
$$

La vérification des équations de la dynamique est équivalente à :

$k^{2} \omega^{2} \rho \underline{W}=k^{2} \omega^{2} \mathbf{K}\left[\underline{W} \underline{P}^{t}\right]_{s y m} \underline{P}-i k \omega\left(\underline{\operatorname{div}} \mathbf{K}\left[\underline{W} \underline{P}^{t}\right]_{s y m}+\mathbf{K} \varepsilon(\underline{W}) \underline{P}\right)-\underline{\operatorname{div}} \mathbf{K} \varepsilon(\underline{W})$ 
ou encore à :

$$
\underline{W}-\frac{\mathbf{A}}{p^{2}} \underline{W}=-\frac{i}{\rho k \omega}\left(\underline{\operatorname{div}}\left(\mathbf{K}\left[\underline{W} \underline{P}^{t}\right]_{s y m}+\mathbf{K} \varepsilon(\underline{W}) \underline{P}\right)-\frac{1}{\rho k^{2} \omega^{2}} \underline{\operatorname{div}} \mathbf{K} \varepsilon(\underline{W})\right.
$$

Si $\underline{W}$ est de degré $n$, le membre de gauche est également de degré $n$. Les deux termes du membre de droite sont respectivement de degré $n-1$ et $n-2$. Aussi, il est aisé d'élaborer une démarche générale et systématique pour construire les rayons complexes d'ordre $n$. A titre d'exemple, construisons les rayons d'ordre 1 qui s'écrivent $a$ priori :

$$
\underline{W}(\underline{X}, \underline{P})=\underline{W}^{0}(\underline{P})+\underline{W^{1}}(\underline{P})
$$

où $\underline{W}^{0}$ est constant et $\underline{W}^{1}$ linéaire par rapport à $\underline{X}$. La vérification par $\underline{W}$ des équations [16] est équivalente à :

$$
\begin{aligned}
& \underline{W^{1}}-\frac{\mathbf{A}}{p^{2}} \underline{W}^{1}=0 \\
& \underline{W^{0}}-\frac{\mathbf{A}}{p^{2}} \underline{W}^{0}=-\frac{i}{\rho k \omega}\left(\underline{\operatorname{div}} \mathbf{K}\left[\underline{W^{1}} \underline{P}^{t}\right]_{s y m}+\mathbf{K}_{\varepsilon}\left(\underline{W^{1}}\right) \underline{P}\right)
\end{aligned}
$$

De la relation [18], on tire que

$$
\underline{W}^{1}=a^{1} \underline{W}_{1}+a^{2} \underline{W}_{2}
$$

où $\underline{W}_{1}$ et $\underline{W}_{2}$ sont des vecteurs propres de $\mathbf{A}$ associés à une direction $\underline{u}$. Poursuivons la construction de $a^{1}$, celle de $a^{2}$ étant similaire. Ainsi, à la direction $\underline{u}$, on a associé $\left(p_{1}, \underline{W}_{1}\right)$ solution du problème aux valeurs propres [14]. Considérons l'équation [19]; sachant que $a^{1}=\underline{\Gamma}^{1} \circ \underline{X}$ où $\underline{\Gamma}^{1}$ est un vecteur constant :

$$
\underline{W}^{0}-\frac{\mathbf{A}}{p_{1}^{2}} \underline{W}^{0}=-\frac{i}{\rho k \omega}\left(\mathbf{K}\left[\underline{\Gamma}^{1} \underline{W}_{1}^{t}\right]_{s y m} \underline{P}+\mathbf{K}\left[\underline{W}^{1} \underline{P}^{t}\right]_{s y m} \underline{\Gamma}^{1}\right)
$$

L'inconnue de l'équation [19] est le vecteur $\underline{W}^{0}$. L'opérateur A étant symétrique, l'équation [19] admet une solution si et seulement si le deuxième membre de [19] est orthogonal au noyau de l'opérateur $\left[\mathbf{I}_{\mathbf{d}}-\frac{\mathbf{A}}{p_{1}^{2}}\right]$ qui est égal à $\left\{\lambda \underline{W}^{1} \mid \lambda \in \Re\right\}$. Il en résulte la condition:

$$
\underline{W}^{1} \circ\left(\mathbf{K}\left[\underline{\Gamma}^{1} \underline{W}_{1}^{t}\right]_{s y m} \underline{P}+\mathbf{K}\left[\underline{W}^{1} \underline{P}^{t}\right]_{s y m} \underline{\Gamma}^{1}\right)=0
$$

qui s'écrit encore :

$$
\underline{\Gamma}^{1} \circ\left(\mathbf{K}\left[\underline{W}^{1} \underline{P}^{t}\right]_{s y m} \underline{W}^{1}\right)=0
$$

On a donc :

$$
\underline{\Gamma}^{1}=\gamma^{1} \underline{N}_{3} \wedge\left(\mathbf{K}\left[\underline{W}^{1} \underline{P}^{t}\right]_{s y m} \underline{W}^{1}\right)
$$

où $\gamma^{1}$ est une constante. $\underline{W}^{1}$ est alors égal, pour $p_{2} \neq p_{1}$ :

$$
\underline{W}^{\prime}=q^{l} \underline{W}_{1}+q^{2} \underline{W}_{2} \quad \text { où }
$$




$$
\begin{aligned}
& q^{1} \text { est arbitraire } \\
& q^{2}\left(1-\frac{p_{2}^{2}}{p_{1}^{2}}\right)=-\frac{i}{\rho k \omega}\left\{\begin{array}{l}
\operatorname{Tr}\left[\mathbf{K}\left[\underline{\Gamma}^{1} \underline{W}_{1}^{t}\right]_{s y m}\left[\underline{P} \underline{W}_{2}^{t}\right]_{s y m}\right]+ \\
\left.\operatorname{Tr}\left[\mathbf{K}\left[\underline{\Gamma}^{1} \underline{W}_{2}^{t}\right]_{s y m}\left[\underline{P} \underline{W}_{1}^{t}\right]_{s y m}\right]\right\}
\end{array}\right.
\end{aligned}
$$

En résumé, les rayons complexes d'ordre 1 sont définis à un rayon complexe d'ordre 0 près; ils dépendent de deux paramètres $\gamma^{1}$ et son homologue $\gamma^{2}$ dans le cas où $p_{2} \geq p_{1}$.

4.2.3. Rayons complexes d'ordre n relatifs à une zone «bord» ou à une zone « coin"

Pour simplifier, nous présentons la démarche pour des rayons complexes d'ordre 0 .

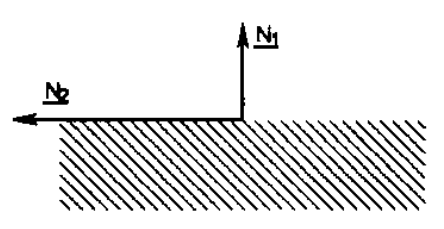

Figure 3. Analyse de vibration de bord

Considérons un bord défini par $X_{1}=0$ [Fig.3], la matière étant contenue dans le demi- espace $X_{1} \leq 0$.

La première caractéristique d'un rayon complexe d'ordre $n$ relatif à la zone bord est d'être admissible pour le milieu semi-infini $\left(X_{1} \leq 0\right)$ et donc de vérifier les équations [11]. Une seconde caractéristique est relative à son expression :

$$
\begin{aligned}
& \underline{U}(\underline{X}, \underline{Y}, \underline{P})=\underline{W}(\underline{X}, \underline{P}) \exp \left(\frac{\delta}{2} \omega \underline{P} \circ \underline{X}\right) \exp \left(\omega P_{1} X_{1}\right) \exp \left(i \omega P_{2} X_{2}\right) \\
& \sigma(\underline{X}, \underline{Y}, \underline{P})=(1+i \eta) \mathbf{K} \varepsilon(\underline{U}) \\
\underline{P}= & -i P_{1} \underline{N}_{1}+P_{2} \underline{N}_{2} \text { où on impose } \\
& -P_{1}>0 \\
& -\underline{W}(\underline{X}, \underline{P}): \text { polynôme de degré } n \text { en la variable lente } \underline{X}\left(\text { ici } X_{2}\right)
\end{aligned}
$$

Les rayons complexes d'ordre 0 sont alors définis par:

$$
\underline{W}=\frac{1}{\rho} \mathbf{K}\left[\underline{W} \underline{P}^{t}\right]_{s y m} \underline{P}
$$

qui se met aussi sous la forme d'un problème aux valeurs propres analogue à [14].

Plutôt que de traiter le cas général, considérons la situation où le matériau est isotrope. On a donc:

$$
\underline{W}=\frac{\gamma}{\rho}(\underline{P} \circ \underline{W}) \underline{P}+\frac{2 \mu}{\rho}\left[\underline{W} \underline{P}^{t}\right]_{s y m} \underline{P}
$$

Il en résulte :

$$
\underline{W}\left(1-\frac{\mu}{\rho}(\underline{P} \circ \underline{P})\right)=\frac{\gamma+\mu}{\rho}(\underline{P} \circ \underline{W}) \underline{P}
$$


Les solutions sont:

où w est un paramètre

$$
\text { - } 1^{\text {er }} \text { Cas }:(\underline{P} \circ \underline{P})=-P_{1}^{2}+P_{2}^{2}=\frac{\rho}{\mu} \quad \underline{W}=w\left(P_{2} \underline{N}_{1}-i P_{1} \underline{N}_{2}\right)
$$

- $2^{\text {ème }}$ Cas $:(\underline{P} \circ \underline{P})=-P_{1}^{2}+P_{2}^{2} \neq \frac{\rho}{\mu} \quad \underline{W}=w \underline{P}$

De [26], on déduit que :

$$
1-\frac{2 \mu+\gamma}{\rho}(\underline{P} \circ \underline{P})=0 \quad \text { et donc }: \quad-P_{1}^{2}+P_{2}^{2}=\frac{\rho}{2 \mu+\gamma} \quad(p 1>0)
$$

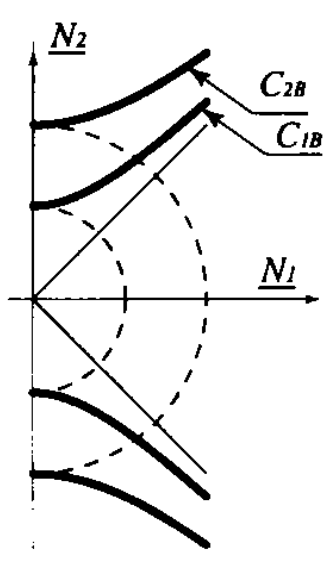

Figure 4. Ensemble des $\left(P_{1}, P_{2}\right)$ admissibles pour une zone «bord»

Ces valeurs $\left(P_{1}, P_{2}\right)$ définissent les courbes $\mathcal{C}_{1 B}$ et $\mathcal{C}_{2 B}$ [Fig.4]; nous ne les retenons pas comme admissibles car les modes correspondants sont plus oscillants que les modes intérieurs. En d'autres termes, nous considérons qu'il n'y a pas de modes «bords ».

Considérons maintenant les rayons complexes d'ordre 0 pour une zone « coin » [Fig.5].

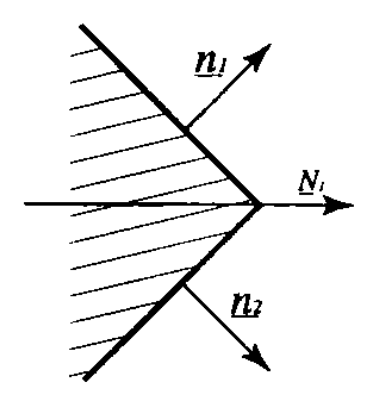

Figure 5. Analyse des vibrations d'une zone "coin»

Le mode de vibration est localisé au voisinage du coin; aussi le vecteur d'onde est assujetti à :

$$
i(\underline{P} \circ \underline{X})=\alpha\left(\underline{n_{1}} \circ \underline{X}\right)+\beta\left(\underline{n_{2}} \circ \underline{X}\right)
$$

avec $\mathcal{R}_{e}(\alpha)>0$ et $\mathcal{R}_{e}(\beta)>0$. Si de surcroît, on considère que les modes réalistes sont tels que $\alpha$ et $\beta$ sont réels, il est aisé de montrer, en écrivant les équations de la 
dynamique, que de tels modes ne peuvent en réalité exister. Dans le cas des plaques, en revanche, il en est autrement: il y a des modes de vibration de «bord " et de " coin ». Pour la situation étudiée ici, on pourra donc se limiter aux modes de vibration relatifs à la zone intérieure.

\subsection{Sous-espaces $\mathrm{S}_{\mathrm{ad}}^{\mathrm{h}}$}

Introduisons des approximations par des rayons d'ordre $n$ tant pour la zone intérieure que pour les zones «bord » et « coin » quand elles existent. Pour la zone intérieure, les rayons d'ordre $n$ forment deux familles:

$-\underline{P} \in \mathcal{C}_{1}$

$$
\begin{aligned}
\underline{U^{1}}(\underline{X}, \underline{Y}, \underline{P}) & =\underline{W}(\underline{X}, \underline{P})\left[\underline{a}^{1}(\underline{P})\right] \exp \left(\frac{\delta}{2} \omega \underline{P} \circ \underline{X}\right) \exp (i \omega \underline{P} \circ \underline{Y}) \\
\sigma^{1}(\underline{X}, \underline{Y}, \underline{P}) & =(1+i \eta) \mathbf{K} \varepsilon\left(\underline{U}^{1}\right) \\
-\underline{P} \in \mathcal{C}_{2} & \\
\underline{U^{2}}(\underline{X}, \underline{Y}, \underline{P}) & =\underline{W}(\underline{X}, \underline{P})\left[\underline{a}^{2}(\underline{P})\right] \exp \left(\frac{\delta}{2} \omega \underline{P} \circ \underline{X}\right) \exp (i \omega \underline{P} \circ \underline{Y}) \\
\sigma^{2}(\underline{X}, \underline{Y}, \underline{P}) & =(1+i \eta) \mathbf{K} \varepsilon\left(\underline{U}^{2}\right)
\end{aligned}
$$

En fait, pour la zone intérieure, on peut prendre en compte toutes les directions possibles de rayon ; la solution correspondante est alors :

$$
\begin{aligned}
\underline{U}^{I}(\underline{X}, \underline{Y}) & =\int_{\mathcal{C}_{1}} \underline{U}^{1}(\underline{X}, \underline{Y}, \underline{P}) d s_{1}+\int_{\mathcal{C}_{2}} \underline{U}^{2}(\underline{X}, \underline{Y}, \underline{P}) d s_{2} \\
\sigma^{I}(\underline{X}, \underline{Y}) & =(1+i \eta) \mathbf{K} \varepsilon\left(\underline{U}^{I}\right)
\end{aligned}
$$

Les intégrations sont menées sur les courbes $\mathcal{C}_{1}$ et $\mathcal{C}_{2}$ définissant l'ensemble des vecteurs d'onde $\underline{P}$ admissibles. Il est clair que $\underline{U}^{I}$ dépend des amplitudes généralisées $\underline{a}^{1}$ et $\underline{a}^{2}$ qui sont des fonctions de $\underline{P}$ définies respectivement sur $\mathcal{C}_{1}$ et $\mathcal{C}_{2}$.

$$
\underline{a}^{1}: \quad \begin{array}{lll}
\underline{P} & \rightarrow \underline{a}^{1}(\underline{P}) \quad \underline{a}^{2}: \quad \frac{P}{\mathcal{C}_{1}} \rightarrow \underline{a}^{2}(\underline{P}) \\
\mathcal{C}_{2} & \rightarrow \mathcal{C}^{m}
\end{array}
$$

Les deux courbes $\mathcal{C}_{1}$ et $\mathcal{C}_{2}$ sont discrétisées par éléments finis. Par exemple, les amplitudes $\underline{a}^{1}$ et $\underline{a}^{2}$ seront prises constantes par élément; on a donc:

$$
\begin{array}{ll}
\underline{a}^{1}(\underline{P})=\mathbf{H}_{1}(\underline{P}) \underline{a}_{1}^{h} & \text { pour } \underline{P} \in \mathcal{C}_{1} \\
\underline{a}^{2}(\underline{P})=\mathbf{H}_{2}(\underline{P}) \underline{a}_{2}^{h} & \text { pour } \underline{P} \in \mathcal{C}_{2}
\end{array}
$$

On note $\underline{a}^{I, h}=\left[\begin{array}{l}\underline{a}_{1}^{h} \\ \underline{a}_{2}^{h}\end{array}\right]$ la colonne des amplitudes généralisées associées à la discrétisation par éléments finis. L'approximation qui en résulte est définie par [30] et [32]; 
on a :

$$
\begin{aligned}
\underline{U}^{I, h}(\underline{X}, \underline{Y}) & =\mathbf{W}^{I, h}\left[\underline{a}^{I, h}\right] \\
\sigma^{I, h}(\underline{X}, \underline{Y}) & =\mathbf{C}^{I, h}\left[\underline{a}^{I, h}\right]
\end{aligned}
$$

où $\mathbf{W}^{I, h}$ et $\mathbf{C}^{I, h}$ sont des opérateurs dépendant de $\underline{X}$ et de $\underline{Y}$. L'amplitude généralisée discrétisée $\underline{a}^{I, h}$ appartient à $\mathcal{C}^{m}$.

Eventuellement pour les zones « bord » et « coin », on opère de manière analogue.

Finalement, en sommant les contributions de la zone intérieure, des zones bords et des zones coins, on obtient :

$$
\begin{aligned}
\underline{U}^{h}(\underline{X}, \underline{Y}) & =\mathbf{W}^{h}\left[\underline{a}^{h}\right] \\
\sigma^{h}(\underline{X}, \underline{Y}) & =\mathbf{C}^{h}\left[\underline{a}^{h}\right]
\end{aligned}
$$

où $\mathbf{W}^{h}$ et $\mathbf{C}^{h}$ sont des opérateurs dépendant explicitement de $\underline{X}$ et de $\underline{Y}$. Le sousespace $\mathbf{S}_{\mathbf{a d}}^{\mathbf{h}}$ (pour $\underline{f_{d}}=0$ ) est alors défini par :

$$
\mathbf{S}_{\mathbf{a d}}^{\mathbf{h}}=\left\{\left(\underline{U}^{h}, \sigma^{h}\right) \quad \mid \quad \underline{U}^{h}=\mathbf{W}^{h}\left[\underline{a}^{h}\right], \sigma^{h}=\mathbf{C}^{h}\left[\underline{a}^{h}\right] ; \underline{a}^{h} \in \mathcal{C}^{k}\right\}
$$

Problème à résoudre pour $\omega$ fixé

Considérons un assemblage de deux sous-structures $S$ et $S^{\prime}$. La formulation variationnelle [4] et les sous-espaces $\mathbf{S}_{\mathbf{a d}}^{\mathbf{h}}, \mathbf{S}_{\mathbf{a d}}^{\mathbf{h}}$ que nous venons d'introduire, conduisent à un système d'équations de dimension $k+k^{\prime}$ sur les complexes:

$$
\left[\mathbf{B}^{h}+\mathbf{Z}^{h}\right]\left[\begin{array}{l}
\underline{a}^{h} \\
{\underline{a^{\prime}}}^{h}
\end{array}\right]=\left[\underline{L}_{d}^{h}\right]
$$

$\mathbf{B}^{h}$ est la matrice d'amortissement; elle est réelle, symétrique et définie positive. L'opérateur $\mathbf{Z}^{h}$ associé à la forme bilinéaire $<\bullet, \bullet>$ est tel que :

$$
\mathbf{Z}^{\mathbf{h}^{t *}}=-\mathbf{Z}^{h}
$$

Des propriétés précédentes, il résulte que l'équation [35] admet toujours une et une seule solution. Le second membre $\underline{L}_{d}^{h}$ dépend des données.

REMARQUE. - En pratique, on peut s'intéresser soit à des excitations associées à des fréquences bien définies, soit à des excitations réparties sur une bande fréquentielle relativement large. Dans ce cas, l'approche présentée pourra être complétée afin de réduire le nombre de résolutions; une technique adaptée sera développée dans un article ultérieur.

\subsection{Grandeurs effectives}

Pour les fréquences moyennes, il ne faut retenir de la solution $\left(s_{h}, s_{h}^{\prime}\right)$ que les grandeurs effectives associées comme par exemple les amplitudes généralisées. La 
distribution spatiale précise de $\left(s_{h}\right.$ et $\left.s_{h}^{\prime}\right)$ n'a pas de sens sur le plan mécanique. Ce point constitue le troisième et dernier principe (P3) qui caractérise la Théorie Variationnelle des Rayons Complexes.

Considérons la sous-structure $S$; les quantités qui interviennent, sont de la forme $q(\underline{X}, \underline{Y})$. Soit L la dimension caractéristique de la cellule d'observation; la valeur effective «surfacique » de $q$ en $\underline{X}$ est alors :

$$
\tilde{q}(\underline{X})=\frac{1}{4 L^{2}} \int_{-L+X_{1}}^{+L+X_{1}} d Y_{1} \int_{-L+X_{2}}^{+L+X_{2}} q(\underline{X}, \underline{Y}) d Y_{2}
$$

Il est également possible de définir une valeur effective « linéique » sur la frontière de $S$. Parmi les quantités effectives intéressantes, on pourra retenir : l'énergie de déformation, l'énergie cinétique, l'intensité vibratoire et la dissipation. Ces quantités tant sur $S$ que sur $\partial S$ pourront être calculées en un certain nombre de points tests. Notons que l'on peut également définir une fenêtre en fréquence. Cela fera l'objet d'un article ultérieur.

\section{Application aux assemblages de plaques en flexion}

Seuls quelques points majeurs sont abordés ; nous renvoyons, pour une vision détaillée, à un article complémentaire.

\subsection{Formulation classique}

Pour simplifier la présentation, on considère l'assemblage de deux plaques isotropes homogènes situées dans un même plan dont les surfaces moyennes sont $\Sigma$ et $\Sigma^{\prime}$ [Fig.6]. La théorie de flexion utilisée est la théorie usuelle de Kirchhoff-Love. Pour la plaque $\Sigma$ (resp. $\Sigma^{\prime}$ ), les quantités pertinentes sont la flèche $w$ (resp. $w^{\prime}$ ) et l'opérateur moment $\mathbf{M}$ (resp. $\mathbf{M}^{\prime}$ ).

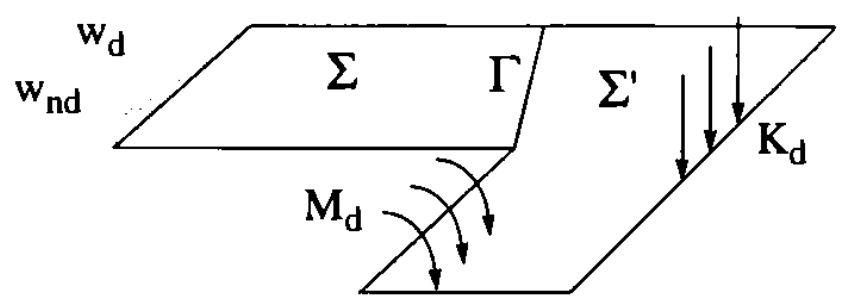

Figure 6. Description de l'assemblage des plaques $\Sigma$ et $\Sigma^{\prime}$ et de leur environnement

On suppose, pour simplifier, que les coefficients d'amortissement $\theta$ et $\theta^{\prime}$ sont nuls. Avec les notations usuelles, le problème de référence à résoudre peut se mettre sous une forme compacte en introduisant les espaces $\mathbf{S}_{\mathbf{a d}}$ et $\mathbf{S}^{\prime}{ }_{\text {ad }}$ relatifs aux sousstructures $\Sigma$ et $\Sigma^{\prime}$. Toutes les quantités considérées sont des amplitudes définies sur les complexes. 
$\mathbf{S}_{\mathbf{a d}}$ est alors défini par:

$$
\begin{aligned}
\mathbf{S}_{\mathbf{a d}}=\{(w, \mathbf{M}) \in \mathcal{U} & \times \mathcal{S} \quad(w, \mathbf{M}) \text { vérifie [38] et [39] }\} \\
\Delta \Delta w \frac{2(1+i \eta) E h^{3}}{3\left(1-\nu^{2}\right)} & =2 \rho h \omega^{2} w \quad \text { sur } \Sigma \\
\mathbf{M} & =\frac{2 h^{3}}{3}(1+i \eta) \mathbf{K}_{c p} \chi(w) \quad \text { sur } \Sigma
\end{aligned}
$$

$\mathbf{S}_{\mathbf{a d}}^{\prime}$ est défini de la même manière. On notera $K_{n}=-(\underline{n} \underline{\operatorname{div}}[\mathbf{M}]+(\underline{t} \mathbf{M} \underline{n}), t)$. Le problème à résoudre s'écrit :

Trouver $(w, \mathbf{M}) \in \mathbf{S}_{\mathbf{a d}}$ et $\left(w^{\prime}, \mathbf{M}^{\prime}\right) \in \mathbf{S}_{\mathbf{a d}}^{\prime}$ tels que les conditions sur le bord et les conditions de transmission soient vérifiées:

$\left.\begin{array}{lllll}w & =w_{d} & \operatorname{sur} \partial_{w d} \Sigma & w & =w^{\prime} \\ w_{, n} & =w_{n d} & \text { sur } \partial_{w n d} \Sigma & w, n & =w_{, n}^{\prime} \\ K_{n} & =K_{d} & \text { sur } \partial_{K d} \Sigma & \underline{n} \mathbf{M} \underline{n} & =\underline{n}^{\prime} \mathbf{M} \underline{n}^{\prime} \\ \|\underline{\mathbf{M}} \underline{n}\| & =0 & \text { points ang. de } \partial \Sigma & K_{n} & =K_{n^{\prime}}^{\prime}\end{array}\right\} \operatorname{sur} \Gamma$

\subsection{La nouvelle Formulation Variationnelle}

Trouver $(w, \mathbf{M}) \in \mathbf{S}_{\mathbf{a d}}$ et $\left(w^{\prime}, \mathbf{M}^{\prime}\right) \in \mathbf{S}_{\mathbf{a d}}^{\prime}$ tels que :

$$
\begin{aligned}
& \mathbf{a}\left(\left[\begin{array}{l}
\delta w \\
\delta w^{\prime} \\
\delta \mathbf{M} \\
\delta \mathbf{M}^{\prime}
\end{array}\right],\left[\begin{array}{l}
w \\
w^{\prime} \\
\mathbf{M} \\
\mathbf{M}^{\prime}
\end{array}\right]\right)-\mathbf{L}\left(\left[\begin{array}{l}
\delta w \\
\delta w^{\prime} \\
\delta \mathbf{M} \\
\delta \mathbf{M}^{\prime}
\end{array}\right]\right)=\mathcal{R}_{e}\{-i \omega[ \\
& \int_{\partial_{w n d} \Sigma} \delta \underline{n} \mathbf{M} \underline{n}\left(w_{, n}-w_{n d}\right)^{*} d L-\int_{\partial_{w d} \Sigma} \delta K_{n}\left(w-w_{d}\right)^{*} d L+ \\
& \int_{\partial_{M d} \Sigma}\left(\underline{n} \mathbf{M} \underline{n}-M_{d}\right) \delta w_{, n}^{*} d L-\int_{\partial_{K d} \Sigma}\left(K_{n}-K_{d}\right) \delta w^{*} d L+ \\
& \int_{\partial_{w n d} \Sigma^{\prime}} \delta \underline{n^{\prime}} \mathbf{M}^{\prime} \underline{n}^{\prime}\left(w_{, n^{\prime}}^{\prime}-w_{n d}^{\prime}\right)^{*} d L-\int_{\partial_{w d} \Sigma^{\prime}} \delta K_{n^{\prime}}^{\prime}\left(w^{\prime}-w_{d}^{\prime}\right)^{*} d L+ \\
& \int_{\partial_{M d} \Sigma^{\prime}}\left(\underline{n}^{\prime} \mathbf{M}^{\prime} \underline{n}^{\prime}-M_{d}^{\prime}\right) \delta w_{, n}^{\prime *} d L-\int_{\partial_{K d^{\prime}} \Sigma^{\prime}}\left(K_{n^{\prime}}^{\prime}-K_{d}^{\prime}\right) \delta w^{\prime *} d L- \\
& \sum_{\text {ang de } \partial \Sigma}\|\underline{\underline{t}} \underline{\mathbf{n}} \underline{\|}\| \delta w^{*}-\sum_{\text {pts ang de } \partial \Sigma^{\prime}}\left\|\underline{t}^{\prime} \mathbf{M}^{\prime} \underline{n}^{\prime}\right\| \delta w^{\prime *}+ \\
& \int_{\Gamma} \frac{1}{2}\left[\delta\left(\underline{n} \mathbf{M} \underline{n}+\underline{n}^{\prime} \mathbf{M}^{\prime} \underline{n}^{\prime}\right)\left(w_{, n}-w_{, n}^{\prime}\right)^{*}-\delta\left(K_{n}+K_{n^{\prime}}^{\prime}\right)\left(w-w^{\prime}\right)^{*}\right] d S+ \\
& \left.\left.\int_{\Gamma} \frac{1}{2}\left[\left(\underline{n} \mathbf{M} \underline{n}-\underline{n}^{\prime} \mathbf{M}^{\prime} \underline{n}^{\prime}\right) \delta\left(w_{, n}+w_{, n}^{\prime}\right)^{*}-\left(K_{n}-K_{n^{\prime}}^{\prime}\right) \delta\left(w+w^{\prime}\right)^{*}\right] d S\right]\right\}
\end{aligned}
$$




\subsection{Approximation}

Les illustrations numériques présentées ci-après utilisent des rayons complexes d'ordre 0 , intérieurs, de bords et de coins. La démarche est évidemment la même que celle présentée au paragraphe 4 . Pour la plaque $(\Sigma)$, un rayon complexe dont l'expression est :

$$
w(\underline{X}, \underline{Y}, \underline{P})=v(\underline{P}) \exp \left(\frac{\eta}{4} \sqrt{\omega} \underline{P} \circ \underline{X}\right) \exp (i \sqrt{\omega} \underline{P} \circ \underline{Y})
$$

est admissible si et seulement si :

$$
\triangle \triangle w \frac{2(1+i \eta) E h^{3}}{3\left(1-\nu^{2}\right)}=2 \rho h \omega^{2} w \quad \text { sur } \Sigma
$$

et donc : $(\underline{P} \circ \underline{P})^{2}=r^{4}$ avec $r^{4}=\frac{3 \rho}{h^{2}} \frac{1-\nu^{2}}{E}$

Ainsi, l'ensemble des vecteurs d'ondes admissibles pour la zone intérieure est associé à un cercle de rayon $r$. Pour les zones « bords » et les zones « coins », on applique également la démarche décrite dans le paragraphe 4 . Tous ces développements seront détaillés dans un article complémentaire. Il en résulte les sous-espaces $\mathbf{S}_{\mathbf{a d}}^{\mathbf{h}}$ et $\mathbf{S}_{\mathbf{a d}}^{\mathbf{h}}$ et le système d'équations associé.

\section{Exemples}

\subsection{Comparaison à une solution exacte - Influence du nombre de ddls}

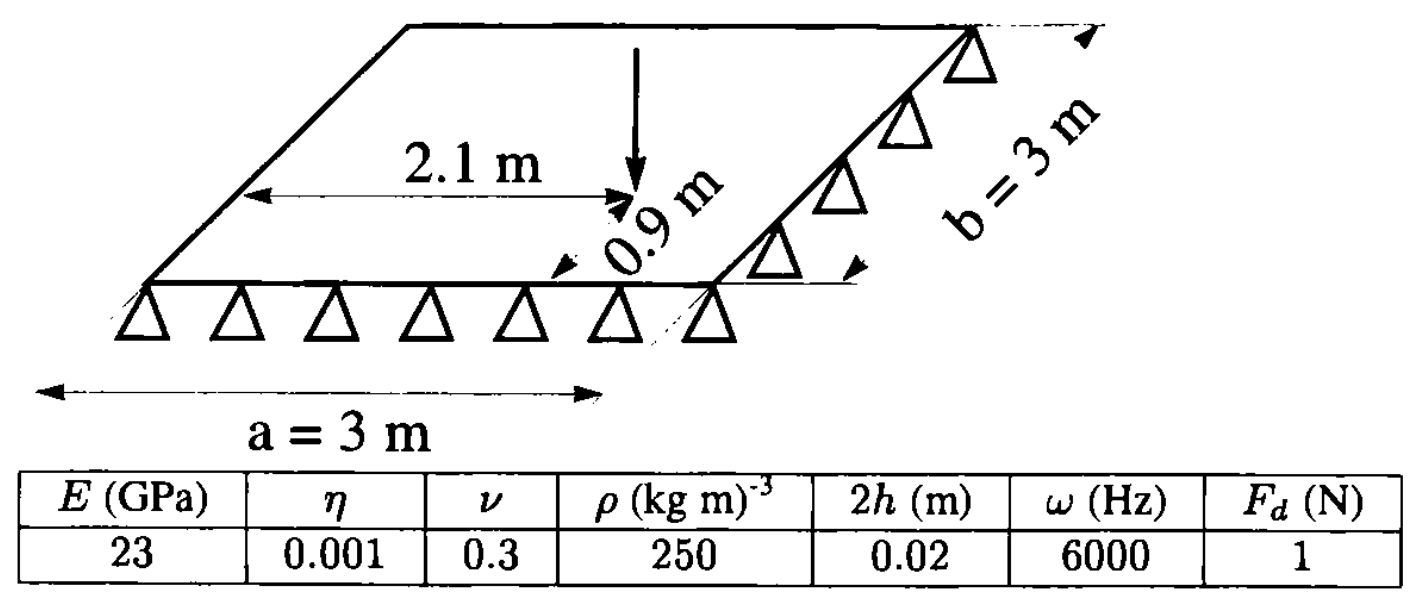

Figure 7. Plaque rectangulaire $\Sigma$ et son environnement

Soit une plaque rectangulaire simplement supportée sur ses côtés, soumise à un effort ponctuel [Fig.7]. Les modes propres de cette structure sont connus analytiquement ; on dispose d'une solution approchée en utilisant les premiers modes. Pour atteindre le domaine des MF, il faut considérer un nombre assez important de modes, de l'ordre de 1000 . La solution, obtenue par superposition des 1600 premiers modes propres, sera prise comme référence [Fig.8]. 


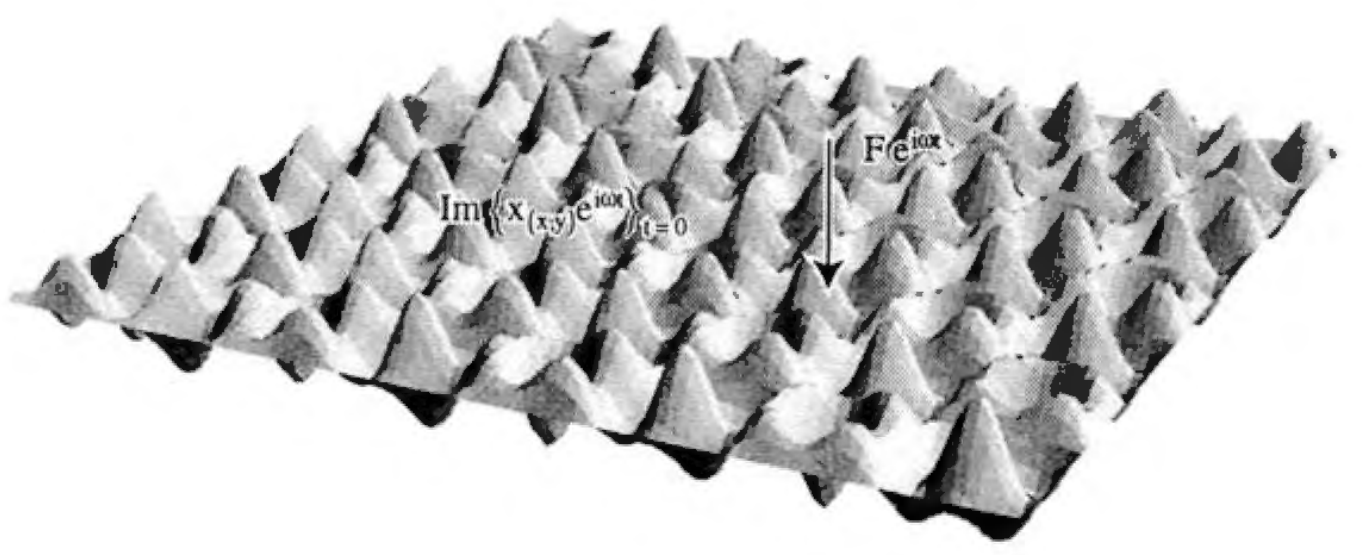

Figure 8. Solution obtenue analytiquement (1 600 modes propres)

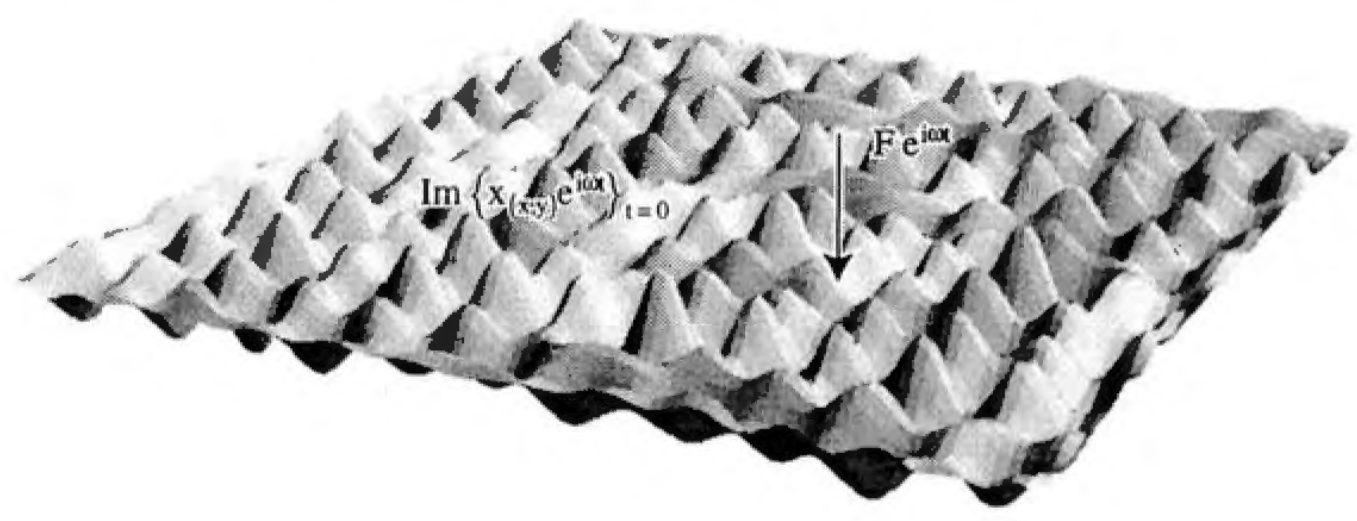

Figure 9. Solution obtenue par la T.V.R.C. (64 DDL)

On introduit dans la Théorie Variationnelle des Rayons Complexes des rayons intérieurs d'ordre 0 , à l'aide des 64 amplitudes généralisées $a_{i}$, ce qui correspond à une discrétisation de la forme :

$$
w(\underline{X})=\sum_{i=1}^{128} a_{i}^{i n t} \int_{\theta=2 \pi \frac{i-1}{128}}^{2 \pi \frac{i}{128}}\left(W \exp \left(\underline{k}^{i n t}(\theta) \underline{X}\right)\right) d \theta
$$

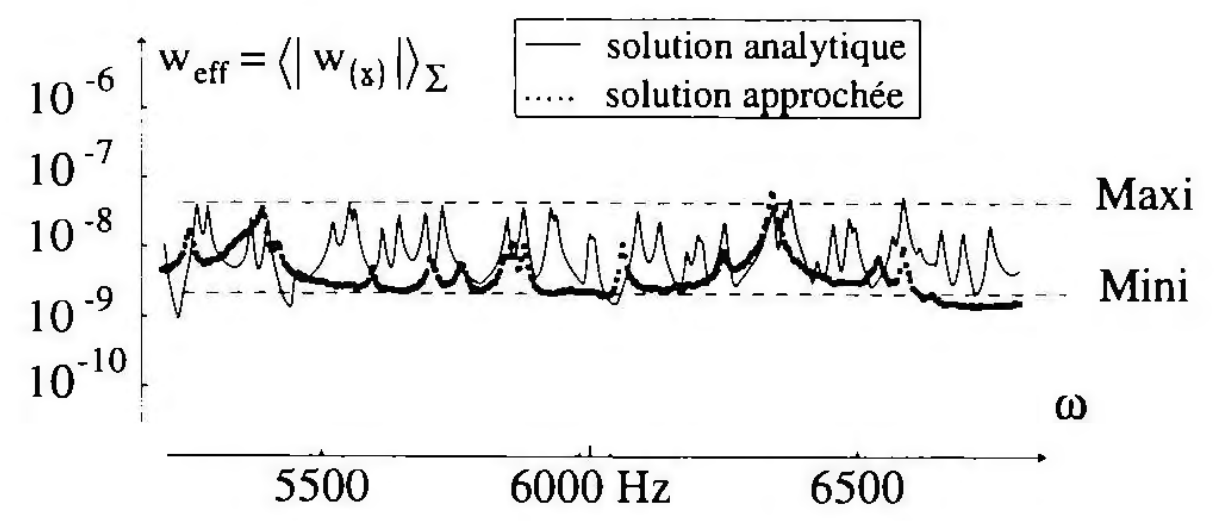

Figure 10. Comparaison sur une bande de fréquence

La solution obtenue par la Théorie Variationnelle des Rayons Complexes est semblable à la solution de référence : l'allure générale est conservée bien que la solution 
puisse être localement différente [Fig.9]. Afin de comparer une grandeur effective, qui seule a un sens, on considère le déplacement moyen de la plaque pour une bande de fréquence autour de $6000 \mathrm{~Hz}$ [Fig.10]. La Théorie Variationnelle des Rayons Complexes permet de prédire un bon ordre de grandeur du niveau effectif mini-maxi sur une bande de fréquence contenant plusieurs modes. Rappelons que ce calcul est fait en n'introduisant que les paramètres définis [Fig.7], et qu'il s'agit bien d'effectuer un calcul prédictif sur les grandeurs effectives.
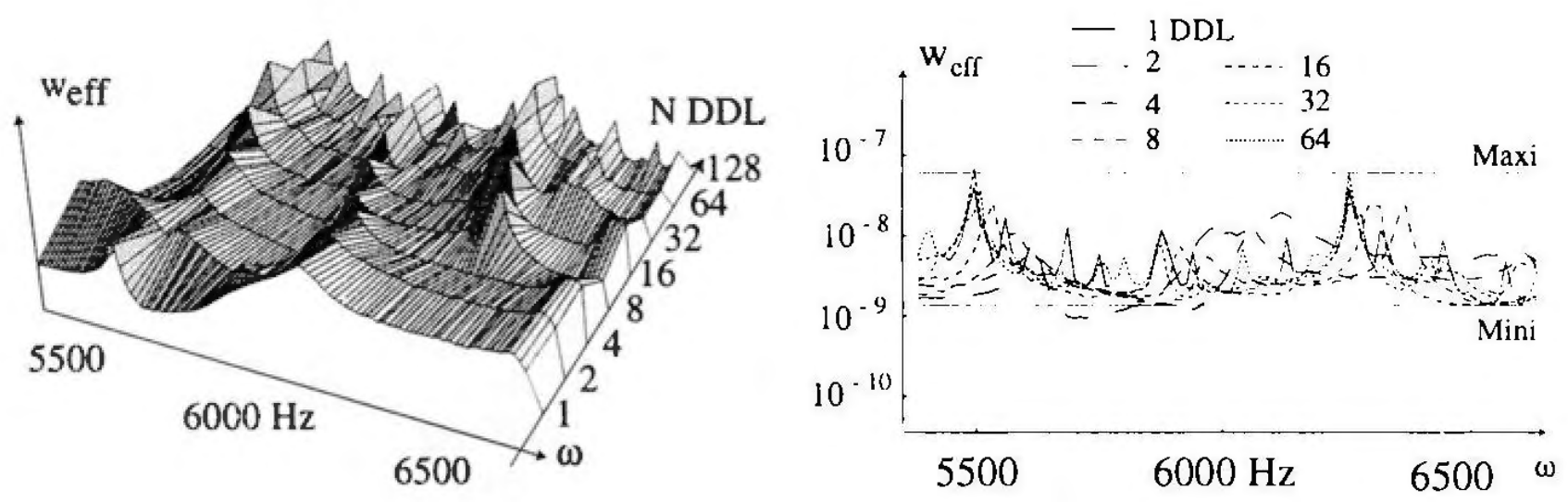

Figure 11. Influence du nombre de $D D L$

Tout en prenant en compte toutes les directions de propagation, on peut choisir le nombre de DDL décrivant la solution. Une première façon de faire varier le nombre de DDL est de représenter des secteurs angulaires plus ou moins larges : il apparaît que le niveau effectif moyen obtenu est peu sensible à la discrétisation choisie (même avec un seul DDL) [Fig.11]. Une discrétisation plus fine permet cependant d'obtenir une information plus riche.

\subsection{Plaque triangulaire}

La Théorie Variationnelle des Rayons Complexes permet d'introduire aussi bien des champs associés à des directions de propagation particulières, que des champs associés à un secteur angulaire. On a préféré une représentation continue permettant d'introduire toutes les directions de propagation sans exception.

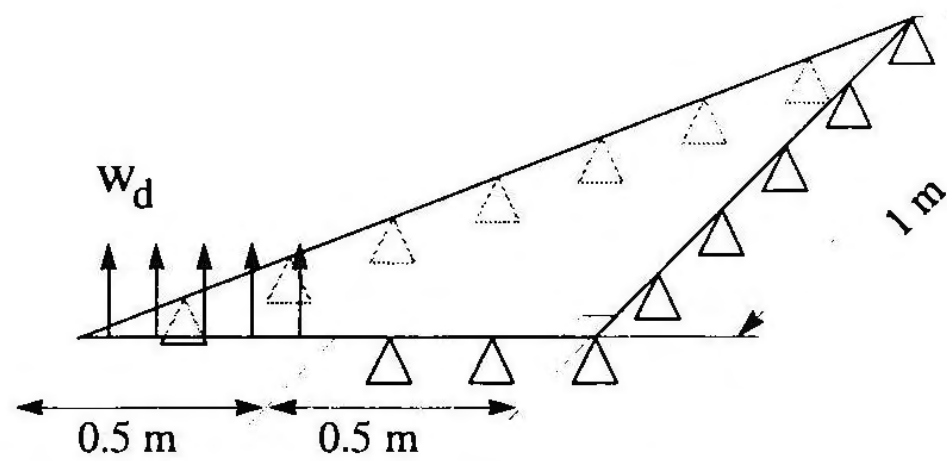

Figure 12. Plaque triangulaire $\Sigma$ et son environnement 
Dans le cas d'une plaque triangulaire [Fig.12], il est pratiquement impossible de prédire quelles sont les directions à introduire. Seule une représentation intégrale des directions assure de n'en oublier aucune. La figure 13 montre le résultat obtenu en considérant 64 DDL pour les rayons intérieurs, 11 DDL par bord et 1 DDL par coin, résultat qui est satisfaisant au regard de la vérification des conditions aux limites.

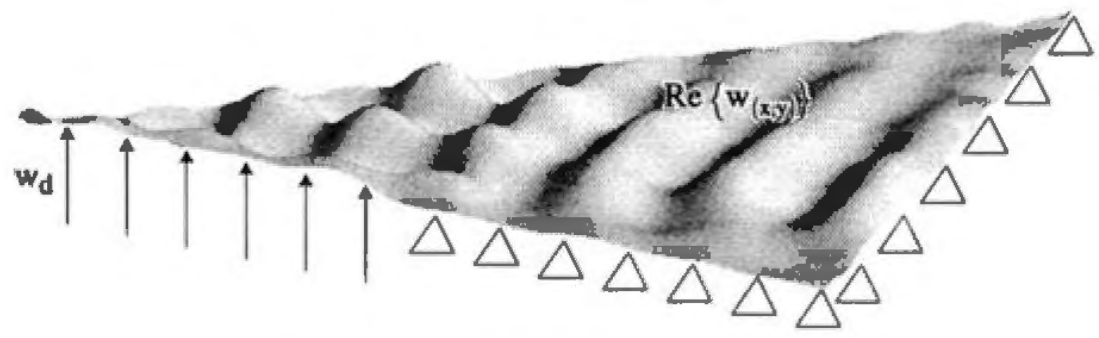

Figure 13. Solution obtenue par F.V.R.C.

\subsection{Assemblage de plaques}

Les assemblages se traitent de la même façon que les autres conditions aux limites, les conditions de transfert sont vérifiées en moyenne au sens de la formulation variationnelle. Aucune condition de compatibilité entre les champs de $\Sigma$ et $\Sigma$ ' n'est exigée. Considérons la structure de la figure 14 : deux plaques $\Sigma$ et $\Sigma$ ' de rigidités différentes assemblées par une interface parfaite.

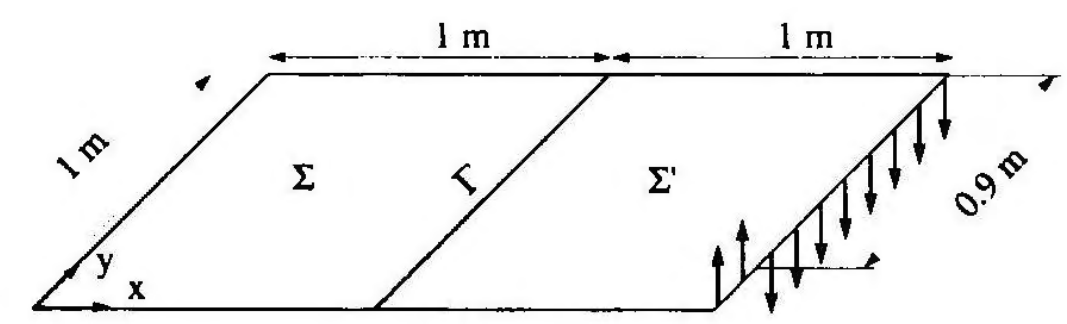

Figure 14. Deux sous-structures et leur environnement

La discrétisation se répartit de la façon suivante: pour $\Sigma$,

- 32 DDL pour les rayons intérieurs de la plaque $\Sigma$ (la plus rigide) et $64 \mathrm{DDL}$

- 9 DDL par bord pour $\Sigma$ et 17 DDL par bord pour $\Sigma$ '.

-1 DDL par coin pour $\Sigma$ et $\Sigma$ '.

Le résultat obtenu est donné sur la figure 15.

La discrétisation au niveau de l'interface n'est pas particulièrement riche et on observe que les conditions d'interface sont vérifiées en moyenne, comme les autres conditions aux limites. Un nombre de sous-structures homogènes quelconque peut être ainsi traité sans difficulté particulière. 


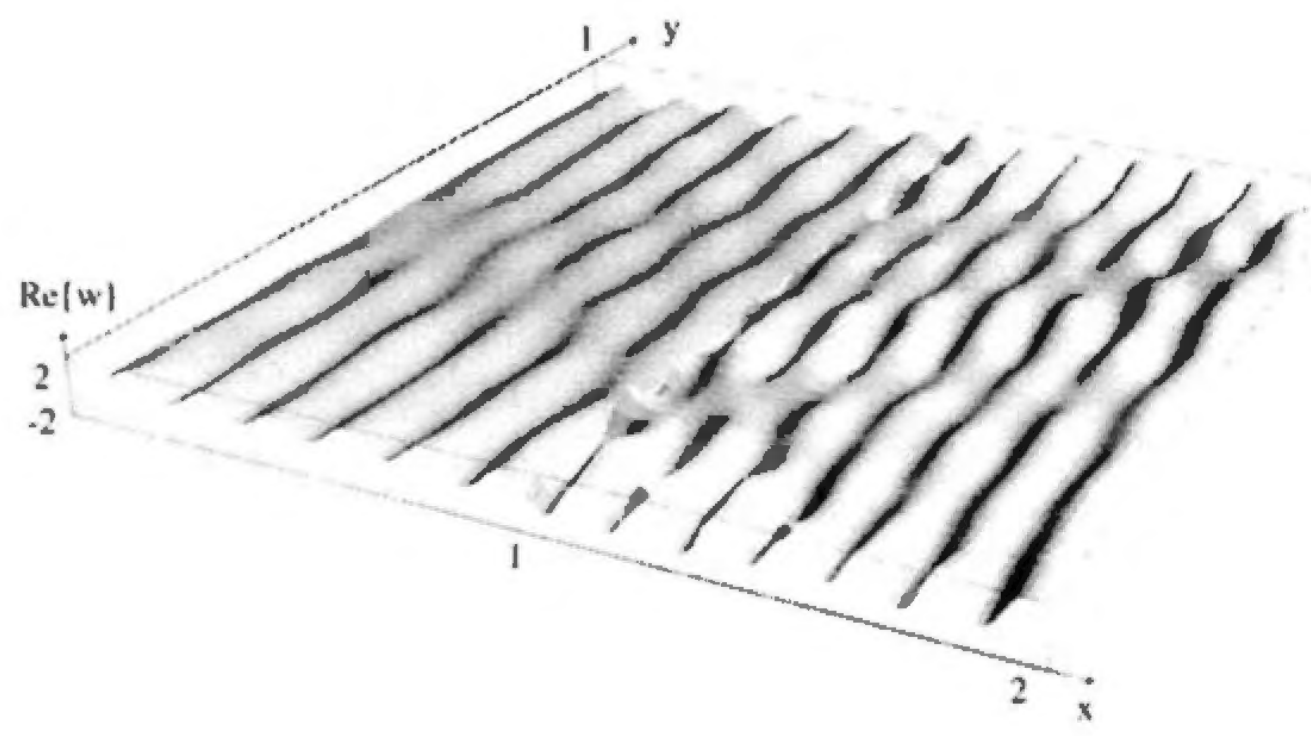

Figure 15. Deux sous-structures de rigidité différente

\section{Conclusion}

L'approche proposée pour calculer les vibrations « moyennes fréquences » appelée Théorie Variationnelle des Rayons Complexes est très générale et repose sur des hypothèses à fort contenu mécanique; dans un certain nombre de situations, comme les assemblages de poutres tant en tension qu'en flexion, elle conduit à la solution exacte. Pour des structures complexes, la TVRC semble être une méthode prometteuse dans le domaine des vibrations moyennes fréquences. Des travaux ultérieurs permettront de mieux cerner son domaine de validité. En particulier, l'application de la théorie aux plaques et aux assemblages de plaques sera détaillée et un traitement approprié des larges bandes de fréquences sera également proposé.

\section{Bibliographie}

[BAB 95] Babuska I., Ihlenburg F., Paikand E. et Sauter S., «A generalized finite element method for solving the Helmotz equation in the two dimensions with minimal pollution », Comput. Methods Appl. Mech. Engrg, vol. 128, p. 325-359, 1995.

[BAR 98] Barbone P., Montgomery J., Michael O. et Harari O., "Scattering by an hybrid asymptotic/finite element method », Comp. Methods Appl. Mech. Engrg, vol. 164, p. 141-146, 1998.

[BEL 75] BELOV V. et RYBACK S., « Applicability of the transport equation in the one dimensional wave propagation problem », Akust. Zh., vol. 21, p. 173-180, 1975.

[BEL 77] BELOV V. et RYBACK S., « Propagation of vibrational energy in absorbing structures ", Journal of Soviet Physics Acoustics, vol. 23/2, p. 115-119, 1977.

[BOU 94] BoUCHE D. et MOLINET F., Méthodes asymptotiques en électromagnétisme. Coll. Mathématiques et applications, Springer, 1994.

[BOU 98] BOUILLARD P. et IHLENBURG F., Error estimation and adaptativity for finite element solution in acoustics in Advances in Adaptative Computational Methods in Mechanics. Ladevèze P., Oden J.T. (eds), Elsevier, 1998. 
[BUV 80] BUVAILO L. et IONOV A., « Application of the finite element method to the investigation of the vibroacoustical characterisics of structures at high audio frequencies », Journal of the Soviet Physics Acoustics, vol. 26 (4), p. 277-279, 1980.

[CUS 90] CUSCHERI J., «Vibration transmission through periodic structures using a mobility power flow approach », Journal of Sound and vibration, vol. 143 (1), p. 65-74, 1990.

[DEM 92] Demkowicz L., Karafiat A. et OdEN J., " Solution of elastic scattering problems in linear acoustics using h-p boundary element method », Comput. Methods Appl. Mech. Eng., vol. 101, p. 251-282, 1992.

[DER 99] Deraemaeker A., Babuska I. et Bouillard P., « Dispersion and pollution of the FEM solution for the Helmholtz equation in one, two and three dimensions », Int. $J$. Numer. Meth. Engng., vol. 46, p. 471-499, 1999.

[DOW 85] DowEll E. et KUBOTA Y., « Asymptotic modal analysis and statistical energy of dynamical systems », Journal of Applied Mechanics, vol. 52, p. 949-957, 1985.

[GIR 93] GIRARD A. et DEFOSSE H., « Frequency response smoothing and structural path analysis: application to beam trusses », Journal of Sound and Vibration, vol. 65 (1), p. 165-170, 1993.

[GRE 99] GREENSTADT J., « Solution of wave propagation problems by the cell discretisation method », Comput. Methods Appl. Mech. Engrg., vol. 174, p. 1-21, 1999.

[GRO 98] GROSH K. et PINSKY P., « Galerkin generalized least square finite element methods for time harmonic structural acoustics », Comp. Methods Appl. Mech. Engrg., vol. 154, p. 299-318, 1998.

[HAR 96] HARARI I. et AAND J.R. HUGES K. G., " Recent developments in finite element methods for structural acoustics », Arch. of Comp. Meth. Eng., vol. 3, p. 131-311, 1996.

[HOC 93] HoChaRd C., LADEVÈze P. et PROSLIER L., " A simplified analysis of elastic structures », Eur. J. Mech. A/Solids, vol. 12(4), p. 509-535, 1993.

[ICH 97] ICHCHOU M., BOT A. L. et JEZEQUEL L., « Energy model of one-dimensional, multipropagative systems », Journal of Sound and Vibration, vol. 201 (5), p. 535-554, 1997.

[ILH 95] ILHENBURG F. et BABUSKA I., « Dispersion analysis and error estimation of Galerkin finite element methods for Helmotz equation », Int. Journ. Num. Meth. Eng., vol. 38, p. 3745-3774, 1995.

[ILH 97] IlHENBURG F. et BABUSKA I., "Finite element solution of th Helmotz equation with high wave number Part 2 : the h-p version of the F.E.M. », SIAM Num. Anal, vol. 34 (1), p. 315-358, 1997.

[LAD 83] LADEVĖZE P., R.D.M. tridimensionnelle, Rapport Interne $N^{\circ} 12$ L.M.T. Cachan, 1983.

[LAD 95] LADEVĖzE P., Prédiction des vibrations moyennes fréquences: Etat de l'art et remarques, Rapport technique, NT Aérospatiale YX/SA $116471,1995$.

[LAD 96a] LADEVĖZE P., «A new computational approach for structure vibrations in the medium frequency range », C. R. Acad. Sci. Paris., vol. t.322, Série II b, n 12, p. 849-856, 1996.

[LAD 96b] LADEVĖZE P., Une nouvelle approche pour le calcul des vibrations moyennes fréquences, Rapport technique, NT Aérospatiale YX/SA 119 639, 1996. 
[LAN 91] LANGRE E. D., Fonctions de transfert de plaques en flexion par équations intégrales. Test de validation et de performance, Rapport technique, CEA :DMT/90/395, 1991.

[LAS 94] LASE Y., ICHCHOU M. et JEZEQUEL L., «Energy flow analysis of bars and beams: theoretical formulation », Journal of Sound and Vibration, vol. 192 (1), p. 2981-3005, 1994.

[LEU 62] LEUNG A. et CHAN J., "Fourrier p-element for the analysis of beams and plates », $J A S A$, vol. 34 (5), p. 623-639, 1962.

[LIU 91] LiU W., Zhang Y. et Ramirez M., « Multiple scale finite element methods », Int. J. Num. Meth. Engng, vol. 32, p. 969-990, 1991.

[LUZ 94] LuZZATO E., EDF Workshop proceeding on methods in medium and high frequency : the alternative to S.E.A, 1994.

[LYO 67] LYON R. et MAIDANICK G., " Power flow between linearly coupled oscillators », $J A S A$, vol. 34 (5), p. 623-639, 1967.

[MAC 94] MACE B., " On the statistical energy analysis hypothesis of coupling power proportionality and some implications of its failure ", JASA, vol. 178 (1), p. 95-112, 1994.

[MOR 92] MORAND J., "A modal hybridization method for the reduction of dynamic models », In New Advances Computational Structural Mechanics, p. 347-365. Ladevèze P., Zienkiewicz O.C. (eds), Elsevier, 1992.

[NEF 89] NEFSKE D. et SUNG S., "Power flow finite element analysis of dynamic systems: basic theory and application of beams », Journal of Vibration, Acoustic, Stress and Reliability in Design, ASME, vol. 111, p. 94-100, 1989.

[OHA 89] OHAYON R., "Local and global effects in the vibration of structures. A review Synthesis ", In ESA Workshop Proceeding on modal representation of flexible structures by continuum methods, Noordwijk (Netherlands), p. 29-54, 1989.

[RIZ 85] Rizzo F., SHIPPY D. et REZAYAT M., « A boudary integral equation method for radiation and scattering of elasic waves in the three dimensions ", Int. J. Num. Meth. Eng., vol. 21, p. 115-129, 1985.

[ROS 97] RoSENhouse G., AVRASHI J. et MiChaEL O., « Steady state elastodynamics using boundary spectral line strips », Engineering computations, vol. 15 (2), p. 221-232, 1997.

[SOI 85] SOIZE C., « The local effects in the linear dynamic analysis of structures in the medium frequency range ", In Local Effects in the Analysis of Structures, p. 253-278. Ladevèze P. (ed), Elsevier, 1985.

[SOI 98] SoIZE C., « Reduced models in the medium frequency range for general dissipative structural-dynamics systems », European Journal of Mechanics A/Solids, vol. 17 (4), 1998.

[WIB 96] WIBERG N., BAUSYS R. et HAGER P., « Improved eigen frequencies and eigenmodes in free vibration analysis », In Advances in Finite Element Technology, p. 43-54. Topping B.H.V. (ed) Civil Comp. Press, 1996.

[WU 98] WU K. et GINSBERG J., « Mid frequency range acoustic radiation from slender elastic bodies using the surface variational principe », Journal of Vibration and Acoustics, vol. 120, p. 392-400, 1998.

[ZIE 87] ZiELINSKI A. et HERRERA I., "Trefftz method: Fitting boudary conditions », Int. J. Num. Meth. Eng., vol. 24, p. 871-891, 1987. 